\title{
Stationary ELM-free H-mode in ASDEX Upgrade
}

\author{
L. Gil ${ }^{1}$, C. Silva ${ }^{1}$, T. Happel ${ }^{2}$, G. Birkenmeier ${ }^{2,3}$, G.D. \\ Conway $^{2}$, L. Guimarais ${ }^{1}$, A. Kallenbach ${ }^{2}$, T. Pütterich ${ }^{2}$, J. \\ Santos $^{1}$, P.A. Schneider ${ }^{2}$, M. Schubert ${ }^{2}$, E. Seliunin ${ }^{1}$, A. Silva ${ }^{1}$, \\ J. Stober ${ }^{2}$, U. Stroth ${ }^{2,3}$, E. Trier ${ }^{2}$, E. Wolfrum ${ }^{2}$, the ASDEX \\ Upgrade Team $\ddagger$ and the EUROfusion MST1 Team $\S$ \\ ${ }^{1}$ Instituto de Plasmas e Fusão Nuclear, Instituto Superior Técnico, Universidade de \\ Lisboa, 1049-001 Lisboa, Portugal \\ 2 Max-Planck-Institut für Plasmaphysik, Boltzmannstr. 2, 85748 Garching, Germany \\ ${ }^{3}$ Physik-Department E28, Technische Universität München, 85748 Garching, \\ Germany \\ E-mail: lgil@ipfn.tecnico.ulisboa.pt
}

February 2020

\begin{abstract}
A stationary H-mode without edge localized modes (ELMs) has been recently achieved in the ASDEX Upgrade tokamak by applying central electron cyclotron resonance heating above the $\mathrm{L}-\mathrm{H}$ power threshold with adequate fueling. It is naturally obtained in favorable $\nabla B$ configuration and has several features which are desirable for future reactors, such as dominant electron heating, low input torque, possibility of access at low input power, good energy confinement $\left(H_{98 \mathrm{y} 2}=0.9-1.3\right)$, high density $\left(f_{\mathrm{GW}}=0.8-0.9\right)$, and no impurity accumulation despite the absence of ELMs. This regime is similar to the EDA H-mode and always features an edge electromagnetic quasi-coherent mode which seems to be responsible for enhanced transport losses, as its appearance and disappearance are correlated with changes in edge and divertor parameters.
\end{abstract}

Keywords: ELM-free, H-mode, quasi-coherent mode, high confinement, EDA, pedestal, ASDEX Upgrade

The H-mode is usually regarded as the preferable operation regime for a fusion reactor due to its superior confinement properties [1], but it comes with a major disadvantage: edge localized modes (ELMs), which limit the achievable edge pressure and lead to unacceptably high heat loads on the divertor plates when extrapolated to large-scale machines [2]. On the other hand, the H-mode requires ELMs for the expulsion of impurities from the plasma. In fact, avoidance of ELMs by poorly heating or fueling the plasma typically results in transient behaviour, often caused by impurity accumulation and possibly leading to radiative collapse, especially in devices with

$\ddagger$ See the author list of H. Meyer et al. 2019 Nucl. Fusion 59112014

$\S$ See the author list of B. Labit et al. 2019 Nucl. Fusion 590860020 
tungsten walls. Even though a few steady-state ELM-free modes of operation with confinement above L-mode levels are known [3, 4], each of them has different drawbacks and the ideal solution to the ELM problem has not yet been identified. Therefore the discovery, study and development of alternative regimes is very important for the success of fusion energy research.

A high confinement ELM-free regime particularly relevant for this work is the EDA H-mode [5], originally found in the Alcator C-mod tokamak by applying ion cyclotron range of frequencies (ICRF) heating after a fresh boronization [6]. This regime does not accumulate impurities and is characterized by a prominent edge fluctuation called the quasi-coherent mode (QCM), which enhances plasma transport [7-9], allowing steady-state operation without ELMs. The EDA H-mode in C-mod has been extensively researched [10] and developed into a performant scenario with important accomplishments, such as the highest volume-averaged core plasma pressure ever achieved in a fusion device [11]. That said, the fact that it has only been documented in C-mod and possibly in JFT-2M [12] limits its physical understanding and extrapolation reliability to large-scale reactors like ITER or DEMO. Obtaining and studying similar regimes in other machines is therefore extremely relevant to the quest for fusion energy, especially since several past attempts in other tokamaks were not able to produce desirable scenarios $[13,14]$.

This letter reports on stationary H-mode plasmas without ELMs recently achieved for the first time in the ASDEX Upgrade (AUG) tokamak by applying central electron cyclotron resonance heating $(\mathrm{ECRH})$ above the $\mathrm{L}-\mathrm{H}$ power threshold with adequate fueling. This regime exhibits several features which are desirable for future reactors [15], such as dominant electron heating, low input torque, possibility of access at low input power, good energy confinement, high density, and no impurity accumulation despite the absence of ELMs. Figure 1 shows an example of such a discharge with constant deuterium fueling and a sequence of ECRH steps. This is a lower single null plasma with the ion $\nabla B$ drift pointing towards the X-point (favourable L-H configuration), toroidal magnetic field of $B_{\mathrm{t}}=-2.5 \mathrm{~T}$, plasma current $I_{\mathrm{p}}=0.8 \mathrm{MA}$, elongation $\kappa=1.7$, average triangularity (mean of lower and upper) $\delta_{\text {avg }}=0.25$, and gas puff rate of $2.6 \times 10^{21} \mathrm{e}^{-} / \mathrm{s}$ after the current ramp-up. As the heating power (figure $1(\mathrm{~d})$ ) is increased, the plasma undergoes several regime transitions indicated by vertical dashed lines: from low confinement (L-mode) to an intermediate I-phase at $2.16 \mathrm{~s}$, followed by an ELMy H-mode at $2.95 \mathrm{~s}$. With another ECRH step the plasma finally enters the regime without ELMs at $3.45 \mathrm{~s}$, as evidenced by the disappearance of the large divertor shunt current spikes in figure 1(c). The transition to this ELM-free H-mode is accompanied by a strong density increase (figure $1(\mathrm{a})$ ), reaching a Greenwald fraction $f_{\mathrm{GW}}=0.8$ (figure 1(b)), and the appearance of an edge instability hereafter referred to as the quasi-coherent mode (figure 1(e)). The stored energy in this phase of the discharge is also significantly higher. The density and radiated power stop increasing after less than a second and the plasma remains stationary for about 10 energy confinement times $\left(\tau_{\mathrm{E}}=0.2 \mathrm{~s}\right)$, with an enhancement factor $H_{98 \mathrm{y} 2}=1$ (figure $\left.1(\mathrm{~b})\right)$, until the end of the 


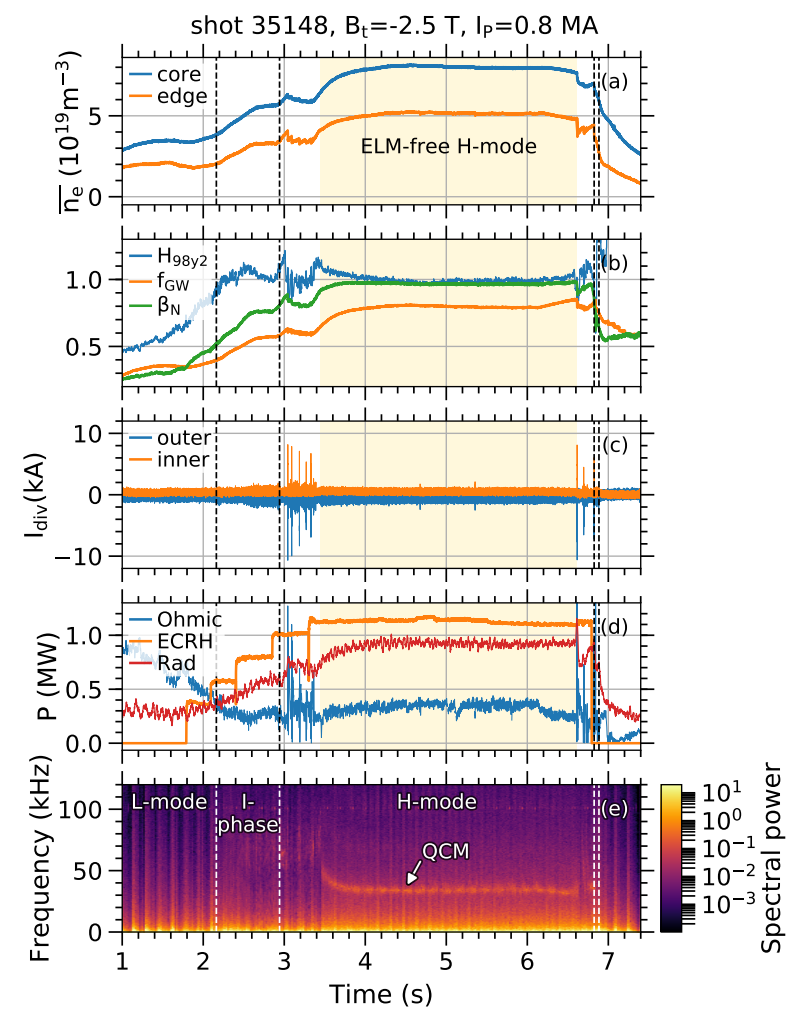

Figure 1. Time evolution of different quantities in a discharge with a stationary ELM-free H-mode: (a) line-averaged electron density, (b) performance indicators (confinement enhancement factor, Greenwald fraction and Troyon-normalized ratio of the plasma pressure to the magnetic pressure), (c) divertor shunt currents as ELM markers, (d) heating and radiated power, and (e) reflectometry phase spectra from edge cutoff layers with electron density $n_{\mathrm{e}}=1.4-2.9 \times 10^{19} \mathrm{~m}^{-3}$. The vertical dashed lines indicate regime transitions.

flattop, being limited in duration only by the inductive current drive.

Varying the plasma current, shape, fueling and heating power, ECRH-only Hmodes without ELMs like the one just described have been achieved in AUG with $H_{98 \mathrm{y} 2}=0.9-1.3, f_{\mathrm{GW}}=0.8-0.9$, and a minimum access power at or slightly above the L-H threshold. These values fit the requirements for ITER [15], making this a very promising mode of operation. The Troyon-normalized plasma pressure, $\beta_{\mathrm{N}}=0.9-1.3$, is slightly lower, and the normalized pedestal collisionality, $\nu_{\mathrm{e}}^{*} \approx 1-2$, and edge safety factor, $q_{95}=4.8-6.9$, so far achieved are still higher than what is predicted for ITER. The extension of these parameters to values closer to those of the ITER baseline scenario will be the subject of future work.

This steady-state ELM-free regime is in fact an H-mode and it possesses an edge transport barrier leading to a pedestal in density, temperature, and therefore pressure, as shown in figure 2. The electron profiles were measured with Thomson scattering (TS) and electron cyclotron emission (ECE) in a discharge with a plasma similar to the one of figure 1. The ECRH power was applied at once instead of slowly increased by steps, resulting in a direct transition from L-mode to the stationary ELM-free H-mode without 


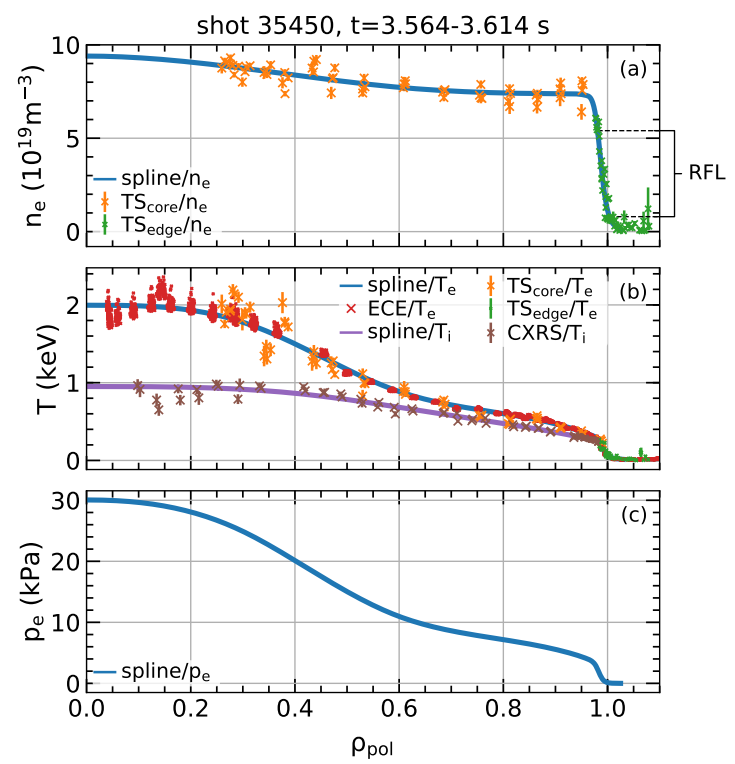

Figure 2. Radial profiles of (a) electron density, (b) electron and ion temperatures and (c) electron pressure during a stationary ELM-free H-mode. The density range covered by the reflectometers which detect the QCM is indicated on the right-hand side of (a).

any ELMs in between, which is an important advantage of this scenario. Neutral beam injection (NBI) blips were then added for charge exchange recombination spectroscopy (CXRS) measurements of the ion temperature. The core density profile is in general flat when compared to the edge, but still with a slight degree of peaking, possibly due to the effect of central ECRH, as is usually observed in ELMy H-mode plasmas [16]. The electron temperature and pressure profiles are peaked due to the central heating. The temperature of the ions in the inner core is lower than that of the electrons because of the pure electron heating but they almost equilibrate in the outer core, for normalized poloidal flux radius $\rho_{\text {pol }}>0.6$. The electron-ion coupling can in principle be further increased by operating at a higher core density.

The ability to maintain a stationary edge pedestal without the relaxation caused by ELMs is a surprising and positive feature of this H-mode. However, large ELMs reappear when the heating power is further increased, as exemplified in figure 3, from 3.95 s onward. Between 3.45 and $3.95 \mathrm{~s}$ the plasma was ELM-free due to the optimal heating power and there was no impurity accumulation. In fact, a strong reduction of the core density of tungsten (the material of the divertor and first wall tiles of AUG), measured by grazing incidence spectroscopy, is observed in this phase (figure $3(\mathrm{c})$ ). Together with the electron density increase, this amounts to a significant reduction of the tungsten concentration, even without ELMs to flush impurities, and enables operation without fresh boronization for wall conditioning. This is likely possible due to a continuous edge transport mechanism, possibly related to the down-chirping QCM visible in the reflectometry spectrogram of figure 3(a).

Adequate fueling is also required to maintain a steady-state ELM-free H-mode. 


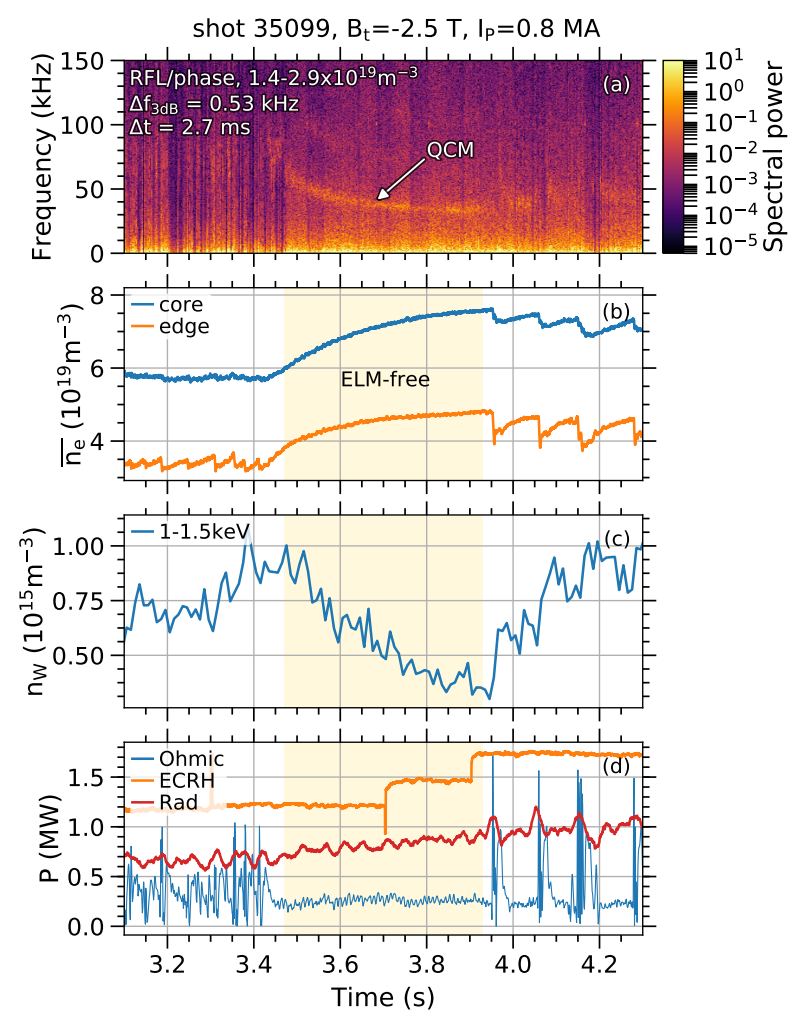

Figure 3. Time evolution of different quantities in a discharge with an ELM-free Hmode period: (a) edge reflectometry phase spectra, (b) line-averaged electron density, (c) core tungsten density, (d) heating and radiated power. The sudden density drops and transient excursions of the ohmic power are due to ELMs.

In fact, when enough ECRH power is applied but the gas puff is too low, the plasma transitions to a non-stationary, impurity-accumulating, ELM-free H-mode without the QCM and whose density and radiated power uncontrollably increase until the occurrence of ELMs and/or radiative collapse. By contrast, changing only the gas puff rate to a moderate value results in a well-behaved stationary ELM-free H-mode with the QCM. It does not accumulate impurities and the radiated power is always below the input power. It is possible to obtain a similar regime with the QCM at higher gas puff levels, but the influence of fueling is not yet fully understood, especially since the access conditions and behavior of the scenario strongly depend also on plasma current, shape and heating power.

The QCM is an electromagnetic instability whose density fluctuations are measured by several diagnostics on the low-field side (LFS), in the steep gradient region of the pedestal and close to the separatrix (see figure 2(a)). So far it has not been possible to precisely measure how far it extends to the pedestal top and whether it exists on the high-field side too. The QCM moves in the electron diamagnetic direction in the lab frame and has a relatively broad frequency peak that gives rise to its name, as shown in the reflectometry and magnetic pickup coils spectra of figure 4 around $33 \mathrm{kHz}$. Its initial frequency at the transition to the ELM-free regime can range from 40 to $80 \mathrm{kHz}$ and then 


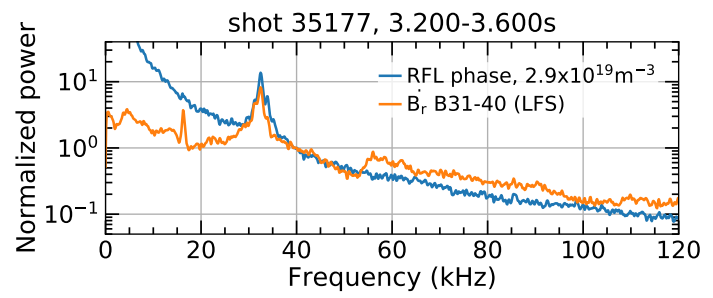

Figure 4. Normalized power spectra of reflectometry phase (blue) caused by density fluctuations and radial magnetic field fluctuations (orange) measured by the magnetic pickup coil closest to the plasma on the LFS.

decreases to a constant value between 20 and $40 \mathrm{kHz}$. The magnetic signature of the QCM is detected only by the pickup coils closest to the plasma, which means that there is a strong radial decay of the magnetic fluctuation amplitude, possibly due to a small perpendicular wavelength. The relation between the two quantities can be derived for a field-aligned perturbation using Laplace's equation in a slab approximation [8]. The pronounced radial decay is consistent with the perpendicular wavenumber of the QCM of $k_{\perp} \approx 0.6 \mathrm{~cm}^{-1} \approx 0.06 \rho_{\mathrm{s}}^{-1}$, as estimated with poloidal correlation reflectometry, where $\rho_{s}$ is the mixed Larmor radius, computed with the electron temperature and ion mass. This corresponds to a toroidal mode number $n \approx 20$, assuming straight magnetic field lines.

In order to further support the idea that the stationary ELM-free H-mode is made possible by enhanced edge transport likely driven by the QCM, it is useful to correlate its appearance with changes in plasma parameters. The discharge shown in figure 5 is particularly suitable for this analysis, because the heating power was kept very close to the lower threshold between ELMy and stationary ELM-free H-mode, resulting in a period of alternation between the two regimes, from 3.33 to $3.55 \mathrm{~s}$. The ELMy Hmode features fast, high frequency edge coherent modes [17], easily detected by the magnetic pickup coils (figure 5(c)), while the stationary ELM-free H-mode features the QCM, with lower velocity, frequency, and wavelength, hardly seen by the coils, but clearly visible for example in an ECE channel close to the separatrix (figure 5(d)). The reflectometer (figure 5(a)) can detect both instabilities, which do not coexist, resulting in the spectral peaks jumping back and forth between low and high frequency. This alternation is correlated with changes in several edge and divertor parameters. Figure 5(e) shows the ECE radiation temperature measured by edge channels which display an oscillation synchronized with the mode alternation. When the QCM appears, the edge line-integrated density and temperature in ECE channels within the confined region decrease, while the temperature and density measured by divertor Langmuir probes increases, as shown in figure 5(f). A rise in the divertor shunt currents is also observed. Low frequency oscillations in the SOL ECE signals and in the poloidal magnetic field close to the divertor are concomitant with these effects. The changes in the divertor happen with a small time delay with respect to the changes in the pedestal, which suggests causality: the QCM appears to cause an increase of particle and energy 


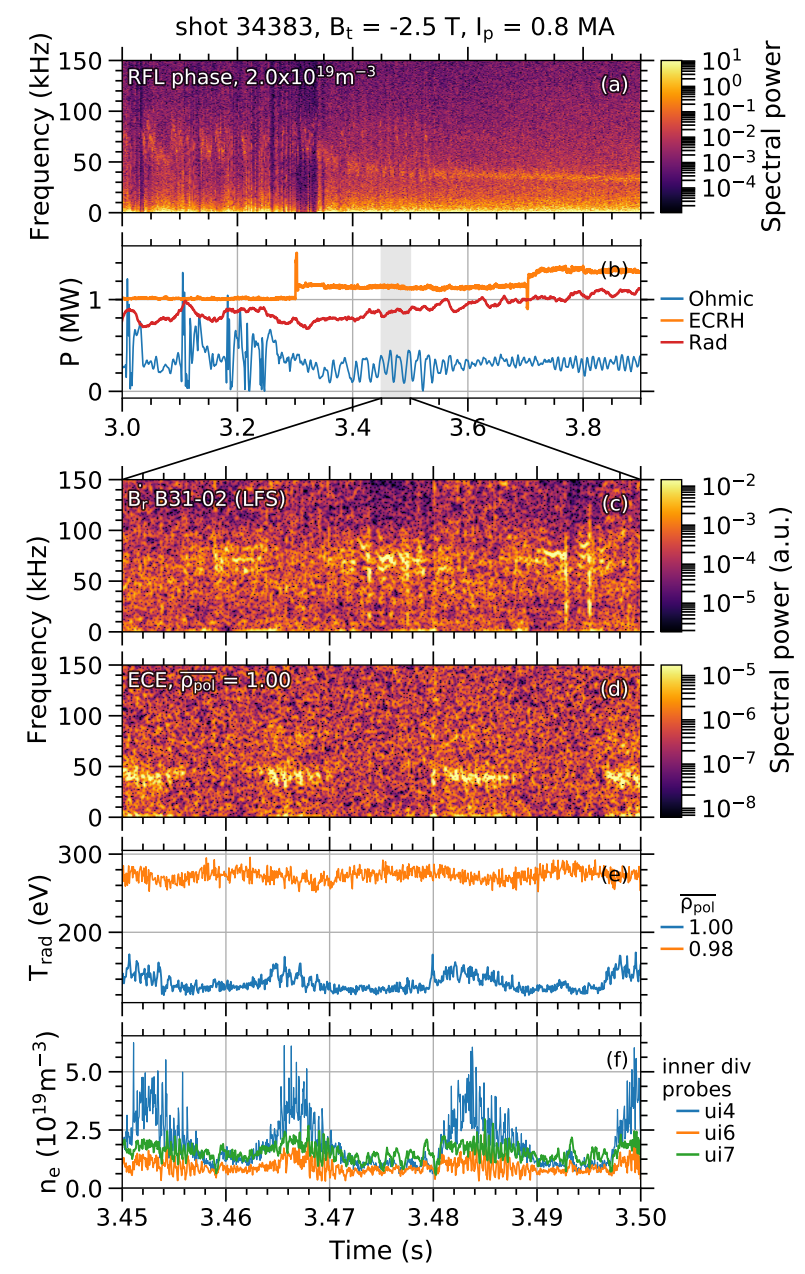

Figure 5. Time evolution of different quantities around the transition to the stationary ELM-free H-mode: (a) edge reflectometry phase spectra, (b) heating and radiated power, (c) LFS radial magnetic field spectra, (d) ECE spectra, (e) ECE radiation temperature, (f) electron density measured by Langmuir probes in the divertor. The time period in the shaded region of panel (b) is zoomed in panels (c)-(f) to highlight the regime alternation.

transport in the pedestal region, expelling plasma to the divertor. This may explain the absence of impurity accumulation and ELMs, as the increased transport could prevent the edge gradients from reaching the peeling-ballooning instability boundary. This type of behavior where an edge instability drives transport in the pedestal or close to the separatrix is a typical feature of high confinement ELM-free regimes in general [3].

Even with the QCM and enhanced transport, large ELMs reappear when the heating power is increased above a certain threshold. At low triangularity, the stationary ELM-free H-mode only exists within a relatively narrow power window of roughly $0.2 \mathrm{MW}$, as previously exemplified in figure 3 . This corresponds to about $20 \%$ of the L-H power threshold, but it is difficult to determine the values precisely, since the minimum ECRH power per heating step in AUG is 0.2 MW. At high triangularity, the situation improves significantly. Figure 6 shows a comparison of two discharges at different 


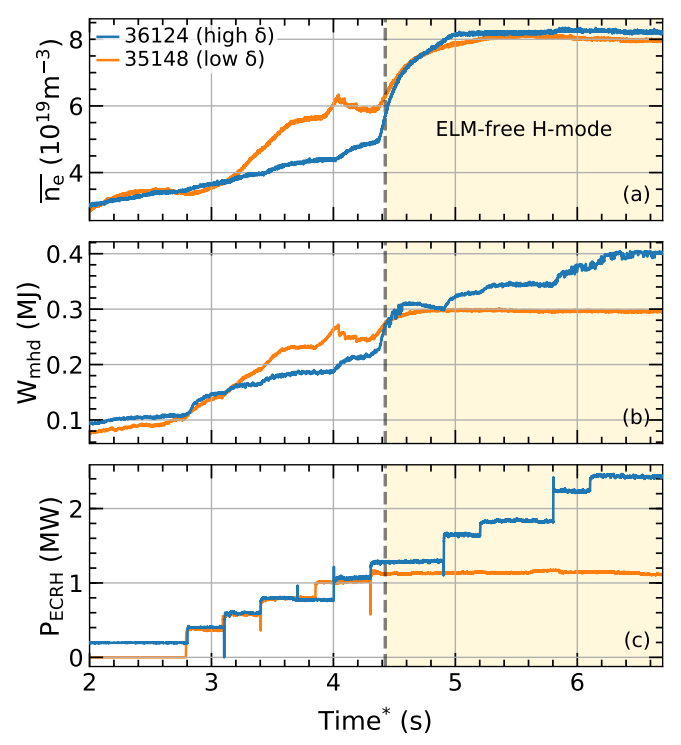

Figure 6. Comparison of low $\delta$ (orange) and high $\delta$ (blue) discharges with a stationary ELM-free H-mode: (a) line-averaged electron density, (b) plasma stored energy, (c) ECRH power. The time axis of the low $\delta$ discharge has been shifted by $1 \mathrm{~s}$ to align the heating ramps of both discharges. The vertical dashed line marks the appearance of the QCM.

triangularity with constant fueling and ECRH steps: the low $\delta$ discharge, in orange, has lower triangularity $\delta_{\text {low }}=0.39$ and upper triangularity $\delta_{\text {up }}=0.12$, corresponding to $\delta_{\text {avg }}=0.25$; the high $\delta$ discharge, in blue, has $\delta_{\text {low }}=0.47, \delta_{\text {up }}=0.30$ and $\delta_{\text {avg }}=0.39$. At low $\delta$, if another ECRH step was applied, large ELMs would occur, but at high $\delta$ the plasma can handle about twice the power while remaining ELM-free. This leads to a much higher core temperature and pressure. A possible explanation for this effect is the extension of the peeling-ballooning stability limit with strong shaping [18]. Another advantage of high $\delta$ operation is that the plasma transitions directly from L-mode to the steady-state ELM-free H-mode, without passing through the ELMy phase that occurs at low $\delta$ with small ECRH steps. As a result, the regime can be accessed within a much wider power window of about $1.2 \mathrm{MW}$, corresponding to $P / P_{\mathrm{LH}} \approx 1-1.8$, where $P$ is the total input power and $P_{\mathrm{LH}}$ is the threshold for the $\mathrm{L}-\mathrm{H}$ transition. This increased robustness at high $\delta$ makes the scenario highly reproducible without requiring a fine control of the input power.

The regime presented in this paper shares several key features with Alcator Cmod's EDA H-mode [5, 6]. It is generally obtained with significant electromagnetic wave heating, becomes more robust at high triangularity [5, 10, 19], does not exist with too low fueling $[5,6]$ and features an edge down-chirping QCM which produces outward plasma transport [7-9], enabling steady-state ELM-free operation with good confinement. Practical differences include the use of ICRF heating in C-mod, compared with ECRH in AUG, and the need of fresh boronization for wall conditioning in C-mod, which is not required in AUG. In terms of dimensionless parameters such as $q_{95}, \beta_{\mathrm{N}}$ and 
$\nu_{\mathrm{e}}^{*}$, the stationary ELM-free H-mode in AUG overlaps with a part of the EDA H-mode parameter space [10], although at a higher $f_{\mathrm{GW}}$ and typically lower $\delta_{\text {avg }}$ than in C-mod. One should note, however, that a large portion of the parameter space for the regime in AUG remains to be explored. Therefore it is possible and even likely that this is a single regime observed in two different machines, which constitutes a powerful opportunity to extend the understanding of its physics and increase the reliability of its extrapolation to large-scale reactors.

To summarize, a stationary ELM-free H-mode with several desirable properties has been recently established and successfully demonstrated in AUG by applying central ECRH above the L-H power threshold with adequate fueling. This low-torque, dominant electron heating regime exhibits good energy confinement, with an enhancement factor $H_{98 \mathrm{y} 2}=0.9-1.3$, high density, with a Greenwald fraction $f_{\mathrm{GW}}=0.8-0.9$, and no impurity accumulation despite the absence of ELMs. It can be accessed at low input power but not too low fueling and becomes more robust at high triangularity, allowing the achievement of higher core temperature and pressure. This promising regime shares several features with the EDA H-mode, including the ubiquitous presence of a QCM which seems to be responsible for enhanced transport losses, as its appearance and disappearance are correlated with corresponding changes in edge and divertor parameters. This enhanced transport may be the key to achieving steady-state operation without ELMs nor impurity accumulation. Additional experiments with argon seeding for pedestal radiative cooling have been started and allowed an increase of the heating power by a factor of two while controlling the net power across the separatrix and maintaining the absence of ELMs. Future studies are planned to widen the parameter space, achieve higher performance, integrate radiative cooling and divertor detachment, derive scalings and further investigate the existence conditions of the QCM as well as its role in regulating edge transport and stability. This will lead to a better understanding of the stationary ELM-free H-mode in AUG, with the ultimate goal of reliably assessing its compatibility with future reactors such as ITER and DEMO.

\section{Acknowledgments}

This work has been carried out within the framework of the EUROfusion Consortium and has received funding from the Euratom research and training programme 20142018 and 2019-2020 under grant agreement No 633053. IST activities also received financial support from "Fundação para a Ciência e a Tecnologia" through project UID/FIS/50010/2019 and grants PD/BD/114326/2016 and PD/00505/2012 in the framework of the Advanced Program in Plasma Science and Engineering (APPLAuSE). The views and opinions expressed herein do not necessarily reflect those of the European Commission. The authors would also like to thank C. Angioni, M. Cavedon, S.S. Denk, J. Hobirk, A. Hubbard, M. Maraschek and F. Ryter for the helpful discussions and advice. 


\section{References}

[1] Wagner F 2007 Plasma Physics and Controlled Fusion 49 B1-B33

[2] Leonard A W 2014 Physics of Plasmas 21090501

[3] Oyama N, Gohil P, Horton L D, Hubbard A E, Hughes J W, Kamada Y, Kamiya K, Leonard A W, Loarte A, Maingi R, Saibene G, Sartori R, Stober J K, Suttrop W, Urano H, West W P and Group t I P T 2006 Plasma Physics and Controlled Fusion 48 A171-A181

[4] Viezzer E 2018 Nuclear Fusion 58115002

[5] Greenwald M, Boivin R, Bonoli P, Budny R, Fiore C, Goetz J, Granetz R, Hubbard A, Hutchinson I, Irby J, LaBombard B, Lin Y, Lipschultz B, Marmar E, Mazurenko A, Mossessian D, Sunn Pedersen T, Pitcher C S, Porkolab M, Rice J, Rowan W, Snipes J, Schilling G, Takase Y, Terry J, Wolfe S, Weaver J, Welch B and Wukitch S 1999 Physics of Plasmas 6 1943-1949

[6] Takase Y, Boivin R L, Bombarda F, Bonoli P T, Christensen C, Fiore C, Garnier D, Goetz J A, Golovato S N, Granetz R, Greenwald M, Horne S F, Hubbard A, Hutchinson I H, Irby J, LaBombard B, Lipschultz B, Marmar E, May M, Mazurenko A, McCracken G, O'Shea P, Porkolab M, Reardon J, Rice J, Rost C, Schachter J, Snipes J A, Stek P, Terry J, Watterson R, Welch B and Wolfe S 1997 Physics of Plasmas 4 1647-1653

[7] Hubbard A E, Boivin R L, Granetz R S, Greenwald M, Hughes J W, Hutchinson I H, Irby J, LaBombard B, Lin Y, Marmar E S, Mazurenko A, Mossessian D, Nelson-Melby E, Porkolab M, Snipes J A, Terry J, Wolfe S, Wukitch S, Carreras B A, Klein V and Pedersen T S 2001 Physics of Plasmas 8 2033-2040

[8] Snipes J A, LaBombard B, Greenwald M, Hutchinson I H, Irby J, Lin Y, Mazurenko A and Porkolab M 2001 Plasma Physics and Controlled Fusion 43 L23-L30

[9] LaBombard B, Golfinopoulos T, Terry J L, Brunner D, Davis E, Greenwald M and Hughes J W 2014 Physics of Plasmas 21056108

[10] Greenwald M, Basse N, Bonoli P, Bravenec R, Edlund E, Ernst D, Fiore C, Granetz R, Hubbard A, Hughes J, Hutchinson I, Irby J, LaBombard B, Lin L, Lin Y, Lipschultz B, Marmar E, Mikkelsen D, Mossessian D, Phillips P, Porkolab M, Rice J, Rowan W, Scott S, Snipes J, Terry J, Wolfe S, Wukitch S and Zhurovich K 2007 Fusion Science and Technology $\mathbf{5 1}$ 266-287

[11] Hughes J, Snyder P, Reinke M, LaBombard B, Mordijck S, Scott S, Tolman E, Baek S, Golfinopoulos T, Granetz R, Greenwald M, Hubbard A, Marmar E, Rice J, White A, Whyte D, Wilks T and Wolfe S 2018 Nuclear Fusion 58112003

[12] Hubbard A E, Kamiya K, Oyama N, Basse N, Biewer T, Edlund E, Hughes J W, Lin L, Porkolab M, Rowan W, Snipes J, Terry J and Wolfe S M 2006 Plasma Physics and Controlled Fusion 48 A121-A129

[13] Mossessian D A, Groebner R J, Moyer R A, Osborne T H, Hughes J W, Greenwald M, Hubbard A and Rhodes T L 2003 Physics of Plasmas 10 689-698 
[14] Stober J, Lomas P J, Saibene G, Andrew Y, Belo P, Conway G D, Herrmann A, Horton L D, Kempenaars M, Koslowski H R, Loarte A, Maddison G P, Maraschek M, McDonald D C, Meigs A G, Monier-Garbet P, Mossessian D A, Nave M F, Oyama N, Parail V, Perez C P, Rimini F, Sartori R, Sips A C and Thomas P R 2005 Nuclear Fusion 45 1213-1223

[15] Doyle E J, Houlberg W A, Kamada Y, Mukhovatov V, Osborne T H, Polevoi A, Bateman G, Connor J W, Cordey J G, Fujita T, Garbet X, Hahm T S, Horton L D, Hubbard A E, Imbeaux F, Jenko F, Kinsey J E, Kishimoto Y, Li J, Luce T C, Martin Y, Ossipenko M, Parail V, Peeters A, Rhodes T L, Rice J E, Roach C M, Rozhansky V, Ryter F, Saibene G, Sartori R, Sips A C, Snipes J A, Sugihara M, Synakowski E J, Takenaga H, Takizuka T, Thomsen K, Wade M R and Wilson H R 2007 Nuclear Fusion 47 S18-S127

[16] Angioni C, McDermott R, Fable E, Fischer R, Pütterich T, Ryter F and Tardini G 2011 Nuclear Fusion 51023006

[17] Medvedeva A, Bottereau C, Clairet F, Conway G D, Manz P, Mink F, Nikolaeva V, Prisiazhniuk D, Stroth U, Wolfrum E, Biancalani A, Heuraux S, Molina D and Silva A 2019 Plasma Physics and Controlled Fusion 61085011

[18] Snyder P B, Wilson H R, Ferron J R, Lao L L, Leonard A W, Osborne T H, Turnbull A D, Mossessian D, Murakami M and Xu X Q 2002 Physics of Plasmas 9 2037-2043

[19] Mossessian D A, Snyder P, Hubbard A, Hughes J W, Greenwald M, LaBombard B, Snipes J A, Wolfe S and Wilson H 2003 Physics of Plasmas 10 1720-1726 